\title{
Bow hunter's syndrome: a sinister cause of vertigo and syncope not to be missed
}

\author{
SC Wong *, TS Chan, CH Chan, Johnny KF Ma
}

Hong Kong Med J 2020;26:150.e1-3

https://doi.org/10.12809/hkmj198048

\section{Case}

In May 2015, a 59-year-old woman presented to Princess Margaret Hospital, Hong Kong, with chronic intermittent vertigo and syncope, aggravated by head rotation to the right. Physical, otoscopy, and nasal endoscopy examination results were unremarkable. Cervical spine plain radiographs demonstrated cervical spondylosis with marginal osteophytosis (Fig 1). In May 2015, computed tomography angiography of the head and neck revealed focal moderate $(50 \%)$ stenosis at bilateral vertebral arteries at $C 5 / 6$ levels due to extrinsic compression from hypertrophied uncovertebral joints (Fig 2). In January 2016, magnetic resonance imaging demonstrated disco-osteophytic protrusion at C5/6 level without evidence of cord compression. In March 2016, digital subtraction angiography, performed with the patient's head in neutral and bilateral rotated positions, demonstrated dynamic deterioration of focal stenosis of right vertebral artery at C5/6 level to up to $80 \%$ stenosis during head rotation to the right (Figs 3 and 4 ).

A static focal moderate $(50 \%)$ stenosis of the left vertebral artery at C5/6 was also present. Overall findings were compatible with bow hunter's syndrome (BHS) with dynamic deterioration of right vertebral artery stenosis on head rotation, related to extrinsic compression by hypertrophied facet joint and disc protrusion. In April 2016, the patient underwent anterior C5/6 cervical discectomy and anterior spinal fusion with smooth recovery and symptomatic resolution.

\section{Discussion}

Bow hunter's syndrome was first reported in 1978 when a patient developed lateral medullary syndrome during archery practice with lateral head rotation. ${ }^{1}$ It refers to symptomatic vertebrobasilar insufficiency secondary to mechanical occlusion or stenosis of vertebral arteries upon head and neck rotation.

The pathogenesis of BHS is related to the tortuous anatomical course of the vertebral artery along the cervical spine, which renders the artery susceptible to extrinsic compression, repetitive shear stress resulting in haemodynamic events in

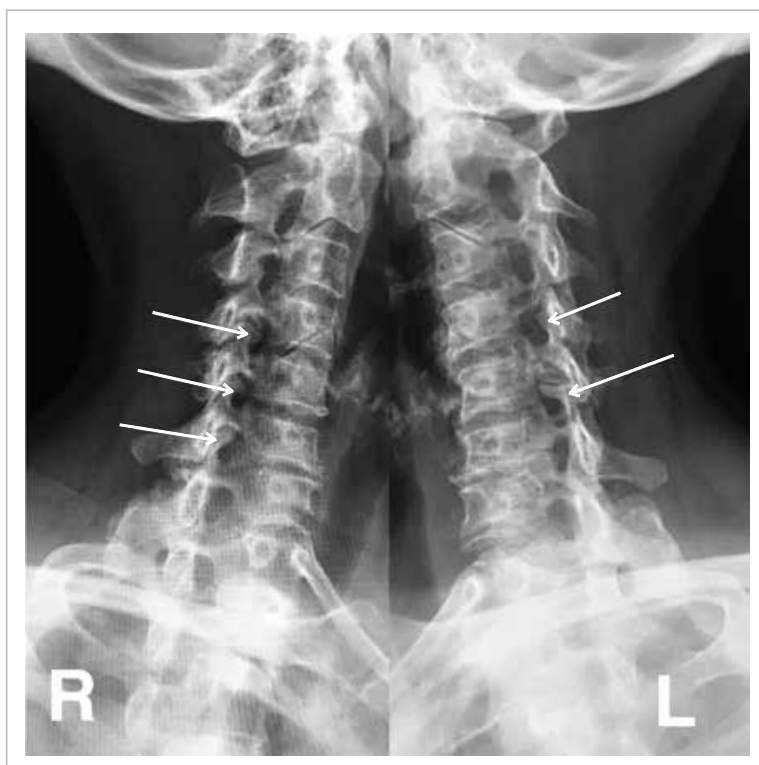

FIG I. Bilateral oblique radiographs of the cervical spine, showing degenerative changes with marginal osteophytes and narrowing of multiple bilateral lower cervical neural foramina (arrows)

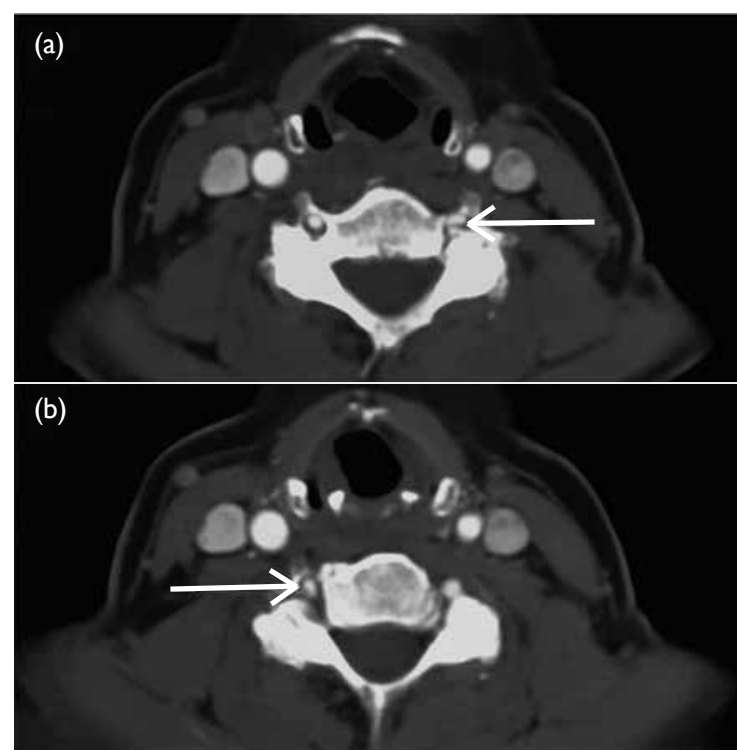

FIG 2. Axial computed tomography angiogram of the bilateral vertebral arteries at $\mathrm{C} 5 / 6$ level with extrinsic compression (arrows) of (a) left and (b) right vertebral artery by hypertrophied uncovertebral joints 


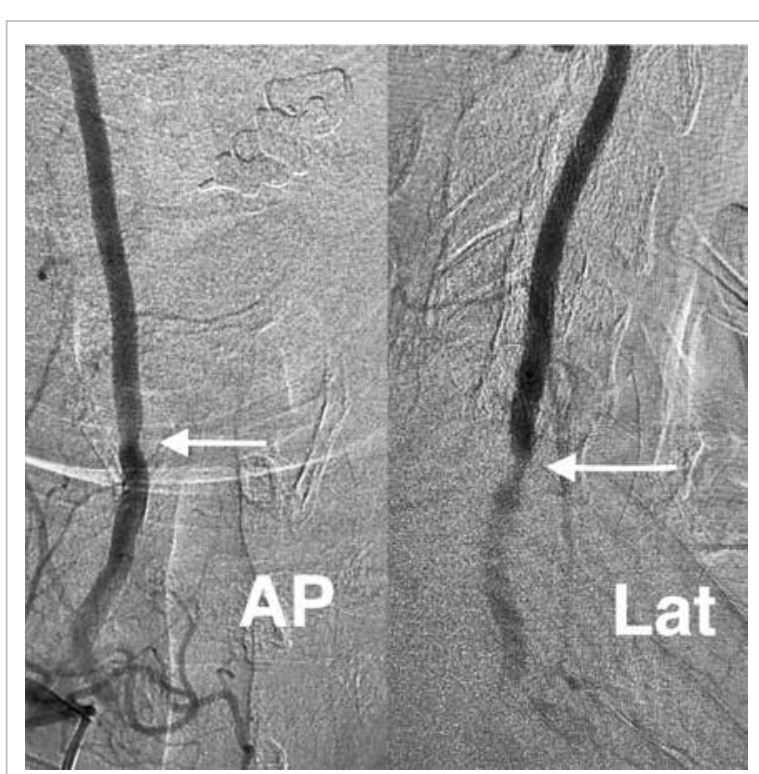

FIG 3. Digital subtraction angiography of the right vertebral artery in frontal (AP) and lateral (Lat) views taken with head in neutral position. Note the focal stenosis of vertebral artery at C5/6 level (arrows)

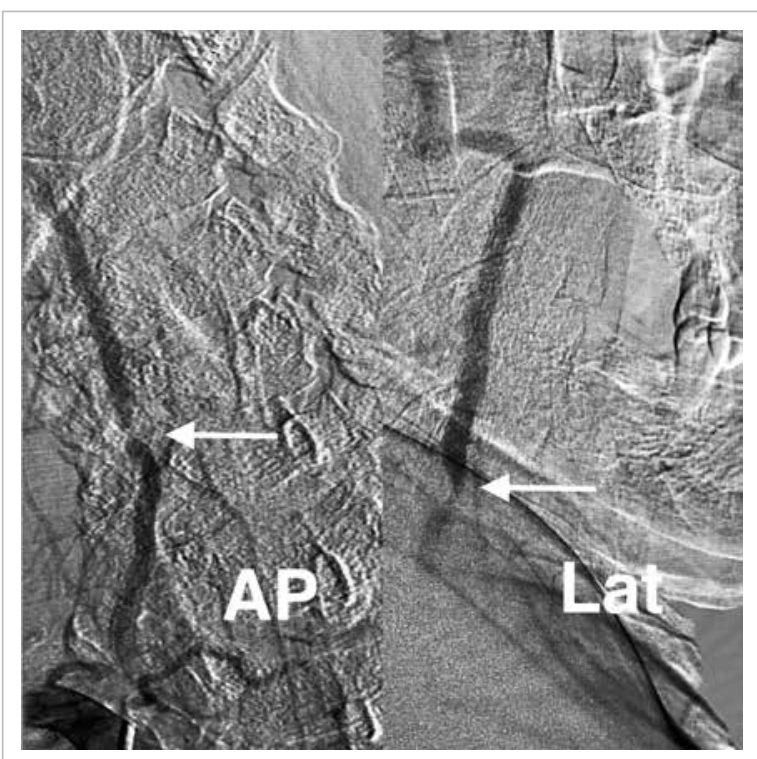

FIG 4. Digital subtraction angiography of the right vertebral artery in frontal (AP) and lateral (Lat) views taken with head rotated to the right. Note the deterioration in vertebral artery stenosis at $\mathrm{C} 5 / 6$ level on head rotation to the right (arrows)

at-risk patients during head and neck rotation. ${ }^{2}$ Osteophytes, disc herniation, ligamentous or neck muscle hypertrophy are risk factors for BHS. ${ }^{3}$

Though rare, BHS is a not to be missed cause of vertigo, owing to its specific relationship with head and neck rotation and its potential risk of posterior circulation ischaemic stroke. ${ }^{1,2}$ Bow hunter's syndrome is more common among males and those aged between 50 and 70 years old. ${ }^{3}$ Common clinical manifestations include vertigo and syncope. Other symptoms include nystagmus, emesis, Horner's syndrome, and rarely motor and sensory deficits. ${ }^{2}$

Imaging is crucial in establishing the diagnosis of BHS, delineating the cause and site of extrinsic compression and evaluating complications such as infarction. Dynamic digital subtraction angiography remains the preferred modality for prompt and accurate localisation of stenotic segment and establishing causal relationship with head rotation. ${ }^{4,5}$ Non-invasive computed tomography or magnetic resonance angiography in both neutral and rotated head positions are also used. Computed tomography can delineate the relationship with surrounding compressive skeletal structures and magnetic resonance would be sensitive in documenting early ischaemic event. $^{3}$

In this case, the patient's complaint of vertigo exacerbation with specific direction of head rotation should raise the suspicion of BHS. Surgical treatment was offered in view of failed conservative approach, repeated fall related to syncope, and underlying uncovertebral joint hypertrophy and disc protrusion as the aetiological factors of vertebral artery compression.

\section{Author contributions}

All authors had full access to the data, contributed to the study, approved the final version for publication, and take responsibility for its accuracy and integrity.

Concept or design: All authors.

Acquisition of data: TS Chan, JKF Ma.

Analysis or interpretation of data: All authors.

Drafting of the manuscript: SC Wong. TS Chan.

Critical revision of the manuscript for important intellectual content: All authors.

\section{Conflicts of interest}

All authors have disclosed no conflicts of interest.

\section{Funding/support}

This pictorial medicine paper received no specific grant from any funding agency in the public, commercial, or not-for-profit sectors.

\section{Ethics approval}

All patients were treated in accordance with the Declaration of Helsinki and provided consent for all investigations and procedures.
SC Wong *, MB, BS
TS Chan, FHKCR, FHKAM (Radiology)
CH Chan, FHKCR, FHKAM (Radiology)
JKF Ma, FRCR (UK), FHKAM (Radiology)

Department of Radiology, Princess Margaret Hospital, Laichikok, Hong Kong

* Corresponding author: bennychun1021@gmail.com 


\section{References}

1. Sorensen BF. Bow hunter's stroke. Neurosurgery 1978;2:259-61.

2. Duan G, Xu J, Shi J, Cao Y. Advances in the pathogenesis, diagnosis and treatment of bow hunter's syndrome: a comprehensive review of the literature. Interv Neurol 2016;5:29-38.

3. Rastogi V, Rawls A, Moore O, et al. Rare etiology of bow hunter's syndrome and systematic review of literature. J Vasc Interv Neurol 2015;8:7-16.

4. Taylor WB 3rd, Vandergriff CL, Opatowsky MJ, Layton KF. Bowhunter's syndrome diagnosed with provocative digital subtraction cerebral angiography. Proc (Bayl Univ Med Cent) 2012;25:26-7.

5. Go G, Hwang SH, Park IS, Park H. Rotational vertebral artery compression: bow hunter's syndrome. J Korean Neurosurg Soc 2013;54:243-5. 